\title{
Prevalence and Causes of Functional Low Vision in School-Age Children: Results from Standardized Population Surveys in Asia, Africa, and Latin America
}

\author{
Clare E. Gilbert, ${ }^{1}$ Leon B. Ellwein, ${ }^{2}$ and \\ the Refractive Error Study in Children Study Group ${ }^{3}$
}

\begin{abstract}
Purpose. Data on the prevalence and causes of functional low vision (FLV) in adults and children are lacking but are important for planning low-vision services. This study was conducted to determine the prevalence and causes of FLV among children recruited in eight population-based prevalence surveys of visual impairment and refractive error from six countries (India [2 locations]; China [2 locations]; Malaysia, Chile, Nepal, and South Africa).
\end{abstract}

Methods. Using the same protocol, 4082 to 6527 children aged 5 (or 7) to 15 years were examined at each site. Uncorrected and presenting visual acuities were successfully measured with retroilluminated logMAR tumbling-E charts in 3997 to 5949 children; cycloplegic autorefraction was performed and best corrected acuities assessed. All children were examined by an ophthalmologist and a cause of visual loss assigned to eyes with uncorrected acuity $\leq 6 / 12$. The prevalence of FLV was determined overall and by site; associations with gender, age, parental education and urban/rural location were assessed with logistic regression.

Results. The prevalence of FLV ranged from 0.65 to 2.75 in 1000 children, with wide confidence intervals. The overall prevalence was 1.52 in 1000 children (95\% CI 1.16-1.95). FLV was significantly associated with age (odds ratio [OR] 1.13 for each year, $P=0.01$ ), and parental education was protective (OR 0.75 for each of five levels of education, $P=0.017$ ). Retinal lesions and amblyopia were the commonest causes.

Conclusions. More studies are needed to determine the prevalence and causes of FLV in children so that services can be planned that promote independence, improve quality of life, and increase access to education. (Invest Ophthalmol Vis Sci. 2008;49:877-881) DOI:10.1167/iovs.07-0973

$\mathrm{D}$ espite there being some population-based data on the prevalence of blindness in children, there are no data on the prevalence and causes of functional low vision in children. These data are urgently needed for rational planning of lowvision services for children.

From the ${ }^{1}$ London School of Hygiene and Tropical Medicine, London, United Kingdom; and the ${ }^{2}$ National Eye Institute, National Institutes of Health, Bethesda, Maryland.

${ }^{3}$ The Study Group members are listed in the Appendix.

Supported by the World Health Organization (WHO) under Contract N01-EY2103 from the National Eye Institute; WHO New Delhi, India; and other nongovernment organizations.

Submitted for publication July 30, 2007; revised September 26, October 19, and November 1, 2007; accepted January 11, 2008.

Disclosure: C.E. Gilbert, None; L.B. Ellwein, None

The publication costs of this article were defrayed in part by page charge payment. This article must therefore be marked "advertisement" in accordance with 18 U.S.C. $\$ 1734$ solely to indicate this fact.

Corresponding author: Clare E. Gilbert, International Centre for Eye Health, Clinical Research Unit, London School of Hygiene and Tropical Medicine, Keppel Street, London WC1E 7HT, UK; clare.gilbert@1shtm.ac.uk.
The World Health Organization's (WHO's) International Classification of Diseases (ICD)-10 categories of visual loss define low vision as "a corrected visual acuity in the better eye of $<6 / 18(<20 / 63)$ down to and including 3/60 (20/400)." This definition includes all individuals, regardless of the cause of visual loss. A major limitation of the ICD-10 categories of visual loss is that they do not allow refractive errors to be assessed as a cause of visual impairment, and so the WHO recently suggested that "presenting visual acuity" (i.e., visual acuity tested with distance spectacles, if usually worn), as well as uncorrected visual acuity, be used in all population-based surveys. Most individuals who have a presenting visual acuity in the better eye of $<6 / 18$ down to and including $3 / 60$ require spectacles, surgery (e.g., cataract surgery), or other treatment to restore sight and thus do not require assessment for lowvision interventions (e.g., optical devices).

At a meeting of low-vision specialists in 1993, it was realized that neither the original ICD-10 definition of low vision nor the revision using presenting acuity adequately identifies individuals who might benefit from low-vision services after assessment. The following definition of low vision was therefore derived for use in population-based prevalence surveys: "a person with low vision is an individual, who after refraction and medical or surgical treatment, has a best corrected visual acuity of $<6 / 18$ to light perception in the better eye, but who uses, or has the potential to use vision for the planning and/or execution of a task." However, it should be acknowledged that others may also have the potential to benefit from low-vision services (e.g., those with better visual acuity but loss of contrast sensitivity). ${ }^{1}$ This definition differs in four ways from the revised ICD-10 definition: (1) best corrected visual acuity rather than presenting vision is used; (2) a broader range of visual acuities is included; (3) individuals whose visual acuity could be improved by surgical and/or medical treatment are excluded; and (4) there is a functional component (e.g., the ability to navigate independently should also be assessed). In this article we use the term "functional" low vision (FLV) to represent the 1993 definition. Data from only two populationbased surveys have been analyzed using the FLV definition to date, one in India $^{2}$ and another in Pakistan ${ }^{3}$ : the former included all age groups and the latter included adults $\geq 30$ years of age.

The purpose of this study was to determine the prevalence and causes of FLV in school-age children who were examined in eight sites in Asia, Africa, and Latin America using the standard methodology developed by the Refractive Error Study in Children (RESC) Group. ${ }^{4}$ The sites were in urban and rural areas: Jhapa District in Eastern Nepal (rural) ${ }^{5}$; Mahabubnagar district near Hyderabad in Southern India (rural) ${ }^{6}$; the Liwan area of Guangzhou, China (urban) ${ }^{7}$; the Shunyi District near Beijing, China (semiurban) ${ }^{8}$; the La Florida area of Santiago, Chile (urban) ${ }^{9}$; the Trilokpuri segment of New Delhi, India (urban) ${ }^{10}$; a contiguous area within the South and West Regions of Durban, South Africa (semiurban/urban) ${ }^{11}$; and the 
Gombak District in Kuala Lumpur, Malaysia (urban). ${ }^{12}$ The surveys were conducted between 1998 and 2003.

Human subject research approval for the RESC study protocol was obtained from the WHO Secretariat Committee on Research Involving Human Subjects. Implementation of the study at each location was approved by the appropriate human subject review committee. The research protocol adhered to the provisions of the Declaration of Helsinki for research involving human subjects.

\section{Methods}

Details of the methods have been published, as have the specific details of each survey. ${ }^{4-12}$

\section{Sample Selection}

Each of the RESC study populations was obtained by random sampling of geographically defined clusters. The originally calculated sample size of 5194 children aged 5 to 15 years (per study site) was based on estimating a prevalence of refractive errors of $22 \%$ within a $20 \%$ error bound with $95 \%$ confidence with upward adjustment to accommodate nonparticipation (10\%) and cluster sampling (25\%). ${ }^{4}$ As there were no reliable population-based data from the study areas that could be used to modify the calculation, this sample size, or larger, was used in planning each survey.

\section{Enumeration}

Using house-to-house visits within the randomly selected clusters, information on the name, age, gender, and schooling of each eligible child, along with the educational level of the parents, was obtained from an interview with an adult family member. Children temporarily absent from the community were included in the enumeration of eligible children, but those away from home for 6 months or more were not. Nonresident visitors were also excluded. In the Mahabubnagar district, Southern India, and the Gombak district, Kuala Lumpur, 5- and 6-year-old children were excluded after pilot exercises demonstrated particular difficulty in obtaining reliable visual acuity measurements among these young children.

\section{Clinical Examination}

Within study sites, clinical examinations were generally performed at one or more locations (e.g., health posts, schools). Written consent for the examination was obtained from a parent or guardian. Presenting and uncorrected monocular visual acuities were measured at $4 \mathrm{~m}$ with a retroilluminated $\operatorname{logMAR}$ chart with five tumbling-E optotypes on each line (Precision Vision, La Salle, IL) and recorded as the smallest line read with one or no errors. After distance visual acuity measurement and evaluation of ocular motility, cycloplegia was induced with 2 drops of $1 \%$ cyclopentolate administered 5 minutes apart, with a third drop after 20 minutes if necessary. Cycloplegia and pupil dilation were evaluated after an additional 15 minutes. Pupillary dilation of 6 $\mathrm{mm}$ or more with an absent light reflex was considered complete cycloplegia. Refractive error was determined by retinoscopy and a handheld autorefractor (Retinomax K-Plus; Nikon, Tokyo, Japan). Best corrected visual acuity was measured after cycloplegic refraction in all children with uncorrected visual acuity of $\leq 6 / 12(\leq 20 / 40)$ in either eye.

The external eye and anterior segment (eyelid, conjunctiva, cornea, iris, and pupil) were examined with a magnifying loupe followed by handheld slit lamp and indirect ophthalmoscopic examination of the media and fundus. The study protocol stipulated that a principal cause of visual impairment be assigned for all eyes with uncorrected visual acuity $\leq 6 / 12$ (from an eight-item checklist), which means that all children defined as having FLV (i.e., best corrected visual acuity of $<6 / 18$ to light perception in the better eye, from untreatable causes) had a cause assigned for each eye. Amblyopia was assigned as the cause of impairment in eyes with no apparent organic lesion and best cor- rected visual acuity $\leq 6 / 12$ if one or more of the following criteria were met: (1) esotropia, exotropia, or vertical tropia at $4 \mathrm{~m}$ fixation, or exotropia or vertical tropia at $0.5 \mathrm{~m}$; (2) anisometropia of 2.00 spherical equivalent diopters or more; or (3) bilateral ametropia of at least +6.00 spherical equivalent diopters. At the discretion of the examiner, eyes that did not meet these explicit criteria were also deemed to have amblyopia as the principal cause of low vision.

\section{Quality Assurance}

Interobserver reproducibility was monitored throughout the course of each study. For visual acuity in right eyes, unweighted $\kappa$ statistics ranged from 0.53 to 0.83 , with $0.0 \%$ to $4.1 \%$ of repeat measurements differing by two or more lines. In left eyes, $\kappa$ statistics ranged from 0.53 to 0.81 with $0.0 \%$ to $3.3 \%$ of measurements differing by two or more lines. Children with uncorrected visual acuity of $\leq 6 / 12$ (in either eye) and approximately $5 \%$ to $10 \%$ of other children had test-retest evaluations of uncorrected visual acuity. The repeat testing was conducted independently by a second examiner who was masked to the findings from the initial testing.

\section{Data Management, Definitions, and Analysis}

Enumeration and examination data forms were reviewed in the field for accuracy and completeness before computer data entry at a central study headquarters at each site. Measurement data ranges, frequency distributions, and consistency among related measurements were checked with data-cleaning programs.

As defined herein, children were considered to have FLV if they had a best corrected visual acuity of $<6 / 18$ down to and including light perception in the better eye and visual loss was not due to a treatable cause in one or both eyes (e.g., cataract). Prevalence rates of FLV were calculated, and associations between FLV and the child's gender, age, parental education (based on the parent with the highest level of schooling and categorized as no education or 1 to 6,7 to 9,10 to 12 , or $>12$ years of schooling), and location (rural, semiurban, or urban) were explored by using logistic regression. Statistical analyses were performed with commercial software (Stata Statistical Software, ver. $\left.8.0^{13}\right)$.

\section{Results}

Across the eight sites, 46,260 children were enumerated, $40,779(87.9 \%)$ of whom were examined (Table 1). Response rates (i.e., the proportion of those enumerated who were examined) ranged from $75.8 \%$ in Santiago, Chile, to $95.9 \%$ in Shunyi, China. In most sites, there was a slight preponderance of boys (range, 49.3\%-51.9\%). Visual acuity measurements were not possible in all children, and visual acuity assessment rates among those with ocular examinations ranged from $91.1 \%$ in the urban site in Delhi to $100 \%$ in the semiurban site in China. Levels of parental education varied across study sites (Table 2). In rural India $86.8 \%$ of parents had received less than 7 years of schooling and only $1.5 \%$ had remained in school for more than 12 years compared with $2.2 \%$ and $12.7 \%$, respectively, in urban China.

A total of 60 children were identified with a best corrected visual acuity of $<6 / 18$, down to and including light perception in the better eye due to untreatable causes. (One child was excluded, as the cause of visual impairment was attributed to malingering.) The overall prevalence of FLV was 1.52 in 1000 (95\% CI: $1.16-1.95 / 1000)$ children, ranging from 0.65 in 1000 children in the urban site in Malaysia to 2.75 in 1000 children in rural southern India. The wide confidence intervals around the estimates reflect the small number of FLV cases (Table 3).

Multivariate analysis showed that the prevalence of FLV was higher in girls than in boys (odds ratio [OR] $1.61 ; 95 \% \mathrm{CI}$ : $0.90-2.98)$, but not at a statistically significant level $(P=0.11)$. FLV increased with age (OR 1.13 for each year of age, $P=$ 
Table 1. Details of Survey Locations, Sample Size, and Gender

\begin{tabular}{|c|c|c|c|c|c|c|c|c|c|}
\hline \multirow[b]{2}{*}{ Country } & \multirow[b]{2}{*}{ Region } & \multirow[b]{2}{*}{ Year } & \multirow{2}{*}{$\begin{array}{l}\text { Age } \\
\text { Group } \\
(y)\end{array}$} & \multirow[b]{2}{*}{ Enumerated } & \multicolumn{2}{|c|}{$\begin{array}{l}\text { Ocular } \\
\text { Examinations }\end{array}$} & \multirow{2}{*}{$\begin{array}{l}\text { Gender } \\
\text { (\% Boys) }\end{array}$} & \multicolumn{2}{|c|}{$\begin{array}{l}\text { Visual Acuity } \\
\text { Measurements }\end{array}$} \\
\hline & & & & & (n) & $(\%)$ & & (n) & $(\%)^{*}$ \\
\hline \multicolumn{10}{|l|}{ Rural locations } \\
\hline India & Andhra Pradesh & $2000 / 01$ & $7-15$ & 4,414 & 4,082 & 92.5 & 51.9 & 3,997 & 97.9 \\
\hline Nepal & Eastern & 1998 & $5-15$ & 5,526 & 5,067 & 91.7 & 51.7 & 4,802 & 94.8 \\
\hline \multicolumn{10}{|c|}{$\begin{array}{l}\text { Urban and semiurban } \\
\text { locations }\end{array}$} \\
\hline India & Delhi (urban) & $2000 / 01$ & $5-15$ & 7,008 & 6,527 & 93.1 & 51.9 & 5,949 & 91.1 \\
\hline Chile & Santiago (urban) & 1998 & $5-15$ & 6,998 & 5,303 & 75.8 & 50.7 & 5,265 & 99.3 \\
\hline Malaysia & Kuala Lumpar (urban) & 2003 & $7-15$ & 5,528 & 4,634 & 83.8 & 51.4 & 4,622 & 99.7 \\
\hline South Africa & Durban (semiurban) & 2002 & $5-15$ & 5,599 & 4,890 & 87.3 & 49.3 & 4,679 & 95.7 \\
\hline China & Shunyi (semiurban) & 1998 & $5-15$ & 6,134 & 5,884 & 95.9 & 51.1 & 5,882 & 99.9 \\
\hline China & Guangzhou (urban) & $2002 / 03$ & $5-15$ & 5,053 & 4,364 & 86.4 & 51.9 & 4,359 & 99.9 \\
\hline & & & & 46,260 & 40,751 & 88.1 & & 39,555 & 97.1 \\
\hline
\end{tabular}

* Proportion of those examined.

$0.01)$ and was inversely associated with parental education (OR 0.75 for each level of education, $P=0.017)$. When included as a covariate, differences between rural, semiurban, and urban locations were not statistically significant.

The causes of low vision are shown in Table 4. Retinal lesions, mainly retinal dystrophies, were the commonest cause, followed by amblyopia. Two children with nonsurgically treated cataract have been included, as both had other ocular conditions likely to result in a poor visual outcome should surgery be performed. There were 11 children with "other" causes: 5 had nystagmus, 1 had coloboma, 2 had unspecified developmental abnormalities, 2 had lesions of the central nervous system, and 1 had an anterior staphyloma (one eye only, with a corneal opacity in the fellow eye). In 11 eyes (6 children) the examining ophthalmologist could not determine the cause of visual loss.

In the FLV study population, 23 children presented with a visual acuity of $<6 / 60$ in the better eye (i.e., severe visual impairment and blindness), which improved to 13 children with best correction (Table 5). The prevalence of severe visual impairment and blindness before and after refraction were therefore 0.58 in 1000 (95\% CI: $0.37-0.87 / 1000)$ and 0.33 in 1000 (95\% CI: $0.18-0.56 / 1000$ ) children, respectively. (The FLV study population does not include one child in New Delhi who had no light perception in both eyes because of bilateral enucleation.)

\section{Discussion}

This article presents the first population-based data on the prevalence and causes of FLV in children. Strengths of this study are that the teams at each survey site used the same definitions, methods, protocols, procedures for quality control, and approaches to data analysis. However, some limitations must be acknowledged. First, the sample size was calculated to determine the prevalence of significant refractive errors, which are much more common than FLV, and the estimates derived are consequently imprecise. Second, the definition of FLV should include an assessment of the individual's ability to function visually, but this was not undertaken, because determining the prevalence and causes of FLV was not a planned outcome of the surveys. Therefore, we may have included a few children with light perception or "hand movements" in the better-seeing eye who would have been excluded if functional vision had been assessed. Last, some children with FLV may not have been enumerated if they were in residential schools for the blind for 6 months or more before the surveys (resulting in an underestimation of FLV). However, in most developing countries, educational provision for children with visual impairment is woefully lacking, with only an estimated $5 \%$ to $10 \%$ of blind children receiving any kind of education. Although not likely, it is also possible that some parents may not have been willing to acknowledge to the enumerators that

TABle 2. Education of Parents, Measured by Years of Schooling of the Parent with the Longest Period at School, in Eight Surveys in Six Countries

\begin{tabular}{|c|c|c|c|c|c|c|c|c|c|c|c|c|}
\hline & \multicolumn{2}{|c|}{ None } & \multicolumn{2}{|c|}{$1-6 y$} & \multicolumn{2}{|c|}{$7-9 y$} & \multicolumn{2}{|c|}{$10-12 y$} & \multicolumn{2}{|c|}{$>12 y$} & \multicolumn{2}{|c|}{ Total $^{*}$} \\
\hline & $n$ & $\%$ & $n$ & $\%$ & $n$ & $\%$ & $n$ & $\%$ & $n$ & $\%$ & $n$ & $\%$ \\
\hline \multicolumn{13}{|l|}{ Rural location } \\
\hline India (AP) & 3,056 & 76.9 & 393 & 9.9 & 167 & 4.2 & 298 & 7.5 & 60 & 1.5 & 3,974 & 100 \\
\hline Nepal & 1,608 & 33.5 & 1,446 & 30.2 & 1,486 & 31.0 & 65 & 1.4 & 188 & 3.9 & 4,793 & 100 \\
\hline \multicolumn{13}{|c|}{$\begin{array}{l}\text { Urban and semiurban } \\
\text { locations }\end{array}$} \\
\hline India (Delhi) & 1,134 & 19.1 & 839 & 14.1 & 1,175 & 19.8 & 1,561 & 26.3 & 1,230 & 20.7 & 5,939 & 100 \\
\hline Chile & 0 & 0 & 2,121 & 43.2 & 2,424 & 49.4 & 360 & 7.3 & 1 & 0.0 & 4,906 & 100 \\
\hline Malaysia & 19 & 0.4 & 411 & 89.7 & 578 & 13.7 & 2,267 & 53.7 & 948 & 22.4 & 4,223 & 100 \\
\hline South Africa & 13 & 0.3 & 626 & 15.3 & 1,181 & 28.9 & 2,223 & 54.4 & 46 & 1.1 & 4,089 & 100 \\
\hline China (S) & 94 & 1.7 & 373 & 6.5 & 3,797 & 66.7 & 1,426 & 25.0 & 6 & 0.1 & 5,696 & 100 \\
\hline China (G) & 5 & 0.1 & 91 & 2.1 & 962 & 22.1 & 2,747 & 63.0 & 553 & 12.7 & 4,358 & 100 \\
\hline Total & 5,929 & 15.6 & 6,300 & 16.6 & 11,770 & 31.0 & 10,947 & 28.8 & 3,032 & 8.0 & 37,978 & 100 \\
\hline
\end{tabular}

* Parental education information was not available for 1577 children, ranging from 1 child in Guangzhou, China to 590 children in Durban, South Africa. 
TABLE 3. Prevalence of FLV in Children Aged 5 or 7 to 15 Years in Eight Surveys in Six Countries

\begin{tabular}{lcccc}
\hline \multicolumn{1}{c}{ Country } & $\begin{array}{c}\text { Visual Acuity } \\
\text { Measurements }\end{array}$ & $\begin{array}{c}\text { FLV } \\
(\boldsymbol{n})\end{array}$ & $\begin{array}{c}\text { Prevalence/1,000 } \\
\text { Children }\end{array}$ & 95\% CI* \\
\hline $\begin{array}{l}\text { Rural locations } \\
\text { India }\end{array}$ & & & & \\
$\quad$ Nepal & 3,997 & 11 & 2.75 & $1.37-4.92$ \\
Urban and semiurban locations & 4,802 & 9 & 1.87 & $0.85-3.55$ \\
$\quad$ India & 5,949 & 14 & 2.35 & $1.29-3.95$ \\
$\quad$ Chile & 5,265 & 11 & 2.09 & $1.04-3.74$ \\
$\quad$ Malaysia & 4,622 & 3 & 0.65 & $0.13-1.90$ \\
South Africa (semiurban) & 4,679 & 4 & 0.85 & $0.23-2.19$ \\
$\quad$ China (semiurban) & 5,882 & 5 & 0.85 & $0.28-1.98$ \\
$\quad$ China & 4,359 & 3 & 0.69 & $0.14-2.01$ \\
Total & 39,555 & 60 & 1.52 & $1.16-1.95$ \\
\hline
\end{tabular}

* Because of prevalences near zero, confidence intervals were calculated by using an exact binomial distribution instead of the normal approximation. Cluster design effects ranging from 0.61 to 1.37 were ignored with the binomial estimates.

they had a visually impaired child, due to the stigma associated with disability.

Reliable population-based data on the prevalence of blindness in children are limited, but the evidence suggests a close association with under-5 mortality rates (U5MRs). ${ }^{14}$ In countries with very low U5MRs (i.e., Western Europe, North America, and the industrialized countries of Southeast Asia), the prevalence of blindness is approximately 0.3 in 1000 children, but can be as high as 1.5 in 1000 children in the poorest countries in Africa and Asia, which have high U5MRs. Indeed, the International Vitamin A Consultancy Group has recently suggested that U5MRs be used as a proxy to indicate whether vitamin A deficiency (a major cause of blindness and mortality in children) is likely to be a public health problem. ${ }^{15}$ In this study the prevalence of FLV also seems to reflect levels of development, as the prevalence was higher in rural areas than in most of the urban areas and lower in countries with higher development indices (e.g., urban China, Malaysia), but differences were not statistically significant. In the multivariate analyses, which pooled data across survey sites, children were significantly less likely to have FLV if their parents were educated, reflecting the findings of many studies of morbidity and mortality in children. ${ }^{16}$

As the number of children identified with FLV in this study is very small, the data on causes should be interpreted with caution, particularly as a cause was not documented in six children. However, children from rural sites were less likely to be amblyopic, possibly reflecting the lower prevalence of refractive errors in children from rural populations compared with urban areas. ${ }^{6,7,11}$ Children in rural locations were more likely to have visual loss from "other" causes, the majority of which were not preventable or treatable.

The pooled data from these surveys seem to suggest that the prevalence of FLV in children is approximately twice the prevalence of severe visual impairment and blindness (using presenting visual acuity in the better eye). This finding is not consistent with the WHO's rule of thumb, which says that $95 \%$ of the number of those who are blind can be used to estimate the number of people with FLV. ${ }^{17}$ However, the confidence intervals around the pooled estimates of FLV as well as severe visual impairment and blindness in our study are wide, which makes these comparisons unreliable.

As provision of low-vision services is a priority of WHO's global initiative, VISION2020 - the Right to Sight, more data would be useful for planners, particularly as children with low vision require frequent and long-term follow-up. Data on the prevalence and magnitude of FLV would also be useful for Ministries of Education and other organizations supporting inclusive education, particularly as the Millennium Development Goal of universal primary education provides an impetus

Table 4. Cause of FLV in Children Aged 5 or 7 to 15 Years in Eight Surveys in Six Countries

\begin{tabular}{|c|c|c|c|c|c|c|c|}
\hline Country & $\begin{array}{c}\text { FLV } \\
\text { Children }\end{array}$ & Amblyopia & $\begin{array}{l}\text { Corneal } \\
\text { Opacity }\end{array}$ & Cataract & $\begin{array}{c}\text { Retinal } \\
\text { Disorder }\end{array}$ & $\begin{array}{l}\text { Other } \\
\text { Causes }\end{array}$ & $\begin{array}{c}\text { Not } \\
\text { Determined }\end{array}$ \\
\hline \multicolumn{8}{|l|}{ Rural locations } \\
\hline Nepal (rural) & 9 & 1 & 0 & 0 & 3 & 4 & 1 \\
\hline India (rural) & 11 & 1 & 2 & 0 & 5 & 3 & 1 \\
\hline \multicolumn{8}{|c|}{ Urban and semiurban locations } \\
\hline India (semiurban) & 14 & 6 & 1 & 0 & 6 & 1 & 0 \\
\hline Chile & 11 & $2^{*}$ & 0 & $1 \dagger$ & 3 & 3 & 4 \\
\hline Malaysia & 3 & 2 & 0 & $1 \neq$ & 0 & 0 & 0 \\
\hline South Africa & 4 & 0 & 1 & 0 & 3 & 0 & 0 \\
\hline China (semiurban) & 5 & 4 & 0 & 0 & 1 & 0 & 0 \\
\hline China (urban) & 3 & 2 & 0 & 0 & 1 & 0 & 0 \\
\hline Total & 60 & 18 & 4 & 2 & 22 & 11 & 6 \\
\hline
\end{tabular}

Data are the number of children in each category. Causes were determined by eye. In 57 children the same cause was recorded in both eyes; in three children there was a different cause in each eye (one child in rural India [corneal opacity and other cause] and two children in Chile [cataract and retinal disorder in one child; amblyopia and undetermined in the other child]).

* Includes one child in whom the diagnosis made in the field was not amblyopia but who had an amblyogenic refractive error in one eye (uncorrected astigmatism of $4.5 \mathrm{D}$ ) with no other disease.

$\dagger$ Nonsurgically treated cataract with high myopia.

‡ Nonsurgically treated cataract with microphthalmos (both eyes). 
TABLE 5. Prevalence of Severe Visual Impairment and Blindness in Children Aged 5 or 7 to 15 Years in Eight Surveys in Six Countries

\begin{tabular}{|c|c|c|c|c|c|c|}
\hline & & \multirow[b]{2}{*}{$\boldsymbol{n}$} & \multicolumn{2}{|c|}{$<6 / 60$ Presenting Acuity } & \multicolumn{2}{|c|}{$<6 / 60$ Best Corrected } \\
\hline & & & $\boldsymbol{n}$ & Prevalence/1,000 & $\boldsymbol{n}$ & Prevalence/1,000 \\
\hline \multicolumn{7}{|l|}{ Rural location } \\
\hline Nepal & Eastern (rural) & 4,802 & 6 & 1.25 & 5 & 1.04 \\
\hline India & Andhra Pradesh (rural) & 3,997 & 6 & 1.50 & 5 & 1.25 \\
\hline \multicolumn{7}{|c|}{$\begin{array}{l}\text { Urban and semiurban } \\
\text { locations }\end{array}$} \\
\hline India & Delhi (urban) & 5,949 & 5 & 0.84 & 2 & 0.34 \\
\hline Chile & Santiago (urban) & 5,265 & 2 & 0.38 & 0 & 0 \\
\hline Malaysia & Kuala Lumpar (urban) & 4,622 & 0 & 0 & 0 & 0 \\
\hline South Africa & Durban (semiurban) & 4,679 & 3 & 0.64 & 0 & 0 \\
\hline China & Shunyi (semiurban) & 5,882 & 1 & 0.17 & 1 & 0.17 \\
\hline China & Guangzhou (urban) & 4,359 & 0 & 0 & 0 & 0 \\
\hline Total & & 39,555 & 23 & 0.58 & 13 & 0.33 \\
\hline
\end{tabular}

and opportunity for improving access to education for visually impaired children in resource-poor countries.

\section{References}

1. World Health Organization. The Management of Low Vision in Children. Report of a WHO Consultation: Bangkok, July 1992. Geneva: World Health Organization, 1993. WHO/PBL/93.27.

2. Dandona R, Dandona L, Srinivas M, Giridhar P, Nutheti R, Rao GN. Planning low vision services in India: a population-based perspective. Ophthalmology. 2002;109:871-878.

3. Shah SP, Minto H, Jadoon Z, et al., on behalf of the Pakistan National Eye Survey Study Group. Prevalence and causes of functional low vision and implications for services: The Pakistan National Blindness and Visual Impairment Survey. Invest Ophthalmol Vis Sci. 2008;49:887-893.

4. Negrel AD, Maul E, Pokharel GP, Zhao J, Ellwein LB. Refractive Error Study in Children: sampling and measurement methods for a multi-country survey. Am J Ophthalmol. 200;129:421-426.

5. Pokharel GP, Negrel AD, Munoz SR, Ellwein LB. Refractive Error Study in Children: results from Mechi Zone, Nepal. Am J Ophthalmol. 2000; $129: 436$ - 444 .

6. Dandona R, Dandona L, Srinivas $\mathrm{M}$, et al. Refractive error in children in a rural population in India. Invest Ophthalmol Vis Sci. 2002; 43:615-622.

7. He M, Zeng J, Liu Y, Xu J, Pokharel GP, Ellwein LB. Refractive error and visual impairment in urban children in southern China. Invest Ophthalmol Vis Sci. 2004;45:793-799.

8. Zhao J, Pan X, Sui R, Munoz SR, Sperduto RD, Ellwein LB. Refractive Error Study in Children: results from Shunyi District, China. Am J Ophthalmol. 2000;129:427-435.

9. Maul E, Barroso S, Munoz SR, Sperduto RD, Ellwein LB. Refractive Error Study in Children: results from La Florida, Chile. Am J Ophthalmol. 129:445454, 2000.

10. Murthy GV, Gupta SK, Ellwein LB, et al. Refractive error in children in an urban population in New Delhi. Invest Ophthalmol Vis Sci. 2002; 43:623-631.
11. Naidoo KS, Raghunandan A, Mashige KP, et al. Refractive error and visual impairment in African children in South Africa. Invest Ophthalmol Vis Sci. 2003;44:3764-3770.

12. Goh PP, Abqariyah Y, Pokharel GP, Ellwein LB. Refractive error and visual impairment in school-age children in Gombak District, Malaysia. Ophthalmology. 2005;112:678-685.

13. Stata Statistical Software: Release 8.0. College Station, TX: Stata Corp.; 2003.

14. Gilbert C, Rahi J, Quinn G. Visual impairment and blindness in children. In: Johnson G, Minassian D, Weale R, West S, eds. Epidemiology of Eye Disease. 2nd ed. London: Edward Arnold Ltd.; 2003:chap 16.

15. Sommer A, Davidson FR. Assessment and control of Vitamin A deficiency: The Annecy Accords. J Nutr. 2002;132: 2845S-2850S.

16. Cochrane SH., Leslie J, O'Hara DJ. Parental education and child health: intracountry evidence. Health Policy Educ. 1982;2:213250.

17. World Health Organization. Asia Pacific regional low vision workshop. Hong Kong, 28-30 May 2001. Geneva: WHO; 2001:PBL/ 02.87 .

\section{Appendix}

\section{RESC Study Group Principal Investigators and Organizational Affiliation during the Survey}

Gopal P. Pokharel, Foundation Eye Care Himalaya, Kathmandu, Nepal; Jialiang Zhao, Peking Union Medical College Hospital, Beijing, China; Eugenio Maul, Pontificia Universidad Catolica de Chile, Santiago, Chile; Lalit Dandona, L. V. Prasad Eye Institute, Hyderbad, India; G. V. S. Murthy, Dr. R. P. Centre for Ophthalmic Sciences, New Delhi, India; Kovin S. Naidoo, University of Durban-Westville, Durban, South Africa; Mingguang He, Zhongshan Ophthalmic Center, Guangzhou, China; Pik-Pin Goh, Hospital Selayang, Selangor, Malaysia. 